\title{
Exercise Dependence Scale: adaptação e evidências de validade e precisão
}

\author{
Exercise Dependence Scale: adaptation and \\ evidences of validity and reliability
}

João Carlos Alchieri', Valdiney Veloso Gouveia², Isabel Cristina Vasconcelos de Oliveira², Emerson Diógenes de Medeiros ${ }^{3}$, Alex Sandro de Moura Grangeiro2 ${ }^{2}$ Carla Fernanda de Lima Santiago da Silva ${ }^{3}$

\section{RESUMO}

Objetivo: O presente estudo teve como objetivo conhecer evidências de validade e precisão da Exercise Dependence Scale-Revised (EDS-R). Métodos: Contou-se com uma amostra não probabilística de 709 praticantes de exercício físico de todo o Brasil, com idades variando entre 12 e 73 anos $(M=25,7$; $D P=8,43$ ), a maioria do sexo masculino $(55,1 \%)$ e apresentando o estado civil solteiro (75,5\%). Os participantes responderam a EDS-R e questões demográficas. Procurando conhecer a estrutura fatorial, foram utilizados procedimentos de análise fatorial exploratória e confirmatória. Adicionalmente, a fim de avaliar a precisão do instrumento, efetuaram-se cálculos de alfa de Cronbach (consistência interna) e correlações de $r$ de Pearson. Resultados: A partir das análises exploratórias (principal axis factoring), foi observada uma variância explicada de 62,7\% para uma estrutura de sete fatores. A consistência interna total da escala foi de 0,88, com índices de alfa de Cronbach variando de 0,68 a 0,89 entre os fatores. Os coeficientes de correlação variaram de 0,10 a 0,65 , apontando para uma inter-relação entre as dimensões. Os procedimentos de análise fatorial confirmatória corroboraram a estrutura heptafatorial a partir de indicadores satisfatórios de bondade de ajuste do modelo $\left[\chi^{2}(168)=288,21 ; \mathrm{p}<0,001, \chi^{2} / \mathrm{gl}=1,78, \mathrm{GFI}=\right.$ $0,93, \mathrm{CFI}=0,95$ e RMSEA $=0,04(\mathrm{IC} 90 \%=0,037-0,055)]$. Conclusões: Os achados apoiaram a adequação psicométrica da EDS-R, a partir das análises exploratórias e confirmatórias, em consonância com o modelo teórico de dependência de exercício físico.

\section{ABSTRACT}

Objective: This study aimed to assess evidence of validity and reliability of Exercise Dependence Scale-Revised (EDS-R). Methods: A non-probabilistic sample consisted of 709 exercise practitioners from all over Brazil, aged 12 to 73 years $(M=25.7 ; S D=8.43)$, most part male (55.1\%) and single (75.5\%). Participants answered EDS-R and demographic issues. About factor structure, procedures of exploratory and confirmatory factor analysis were used. Additionally, to assess measurement reliability, Cronbach's alpha were calculated (internal consistency) and r Pearson correlations. Results: Exploratory analysis (principal axis factoring) suggested an explained variance of $62.7 \%$ for a seven-factor structure. Total

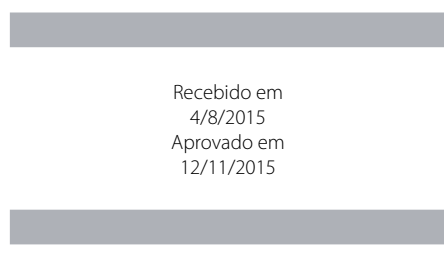

DOI: $10.1590 / 0047-2085000000090$
1 Universidade Federal do Rio Grande do Norte (UFRN). 2 Universidade Federal da Paraíba (UFPB). 3 Universidade Federal do Piauí (UFPI).

Endereço para correspondência: Alex Sandro de Moura Grangeiro Universidade Federal da Paraíba, João Pessoa, PB, Brasil. E-mail:alexsmgrangeiro@gmail.com 


\section{Keywords}

Dependency, exercise, validation studies, psychometric parameters. internal consistency of the scale was 0.88 , with Cronbach's alpha indices ranging from 0.68 to 0.89 among the factors. The correlation coefficients ranged from 0.10 to 0.65 , supporting interrelated dimensions. Confirmatory factor analysis corroborated a seven-factor structure, based on satisfactory indicators of model goodness of fit $\left[\chi^{2}(168)=288.21 ; p<0.001, \chi^{2} / d f=\right.$ 1.78, $\mathrm{GFI}=0.93, \mathrm{CFI}=0.95$ and RMSEA $=0.04(90 \% \mathrm{Cl}=0.037$ to 0.055$)]$. Conclusions: These findings supported the psychometric adequacy of EDS-R, according exploratory and confirmatory analyzes, in line with the theoretical model of exercise dependence.

\section{INTRODUÇÃO}

A dependência de exercício físico (DEF) tem sido abordada por diferentes perspectivas ao longo dos anos ${ }^{1,2}$, variando em função da forma como os autores percebem as consequências da prática de exercícios físicos. Inicialmente, a dependência de exercícios era entendida como positiva (positive addiction), referindo-se às alterações físicas e psicológicas benignas promovidas pela prática regular de exercício físico (EF). Não obstante, posteriormente, com o entendimento de que a prática excessiva de exercícios pode estar associada a aspectos prejudiciais, à saúde física e mental dos indivíduos, passou-se a popularizar a dependência de exercício como algo danoso (negative addiction).

Na literatura nacional, apesar de estar ganhando visibilidade com temas como vigorexia e sua associação aos transtornos alimentares, existem poucos artigos teóricos e estudos que abordem a dependência de exercício ${ }^{2-5}$. Em âmbito internacional, por outro lado, o tema é abordado sistematicamente em pesquisas ${ }^{1,6-9}$, aparecendo associado a outros temas, a exemplo dos transtornos alimentares, imagem corporal, ansiedade e conflitos, comprometimento e paixão por atividade física, além de características de personalidade e diferenças de gênero.

No que consiste, especificamente, à dependência de exercício, pode-se considerá-la como um processo pelo qual a pessoa se sente compelida a exercitar-se ${ }^{10}$, podendo apre-

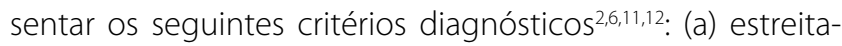
mento do repertório de atividades diárias, levando a um padrão estereotipado de exercícios, uma ou mais vezes por dia; (b) saliência do comportamento de praticar exercícios, dando prioridade sobre outras atividades, para que seja mantido o padrão de exercícios; (c) aumento na tolerância à quantidade e frequência dos exercícios com o decorrer do tempo; (d) sintomas de abstinência relacionados a transtornos de humor (por exemplo, irritabilidade, depressão, ansiedade), quando interrompida a prática de exercícios; (e) alívio ou prevenção do aparecimento de síndrome de abstinência por meio da prática de mais exercícios; ( f) consciência subjetiva da compulsão pela prática de exercícios; e, por fim, (g) rápida reinstalação dos padrões prévios de exercícios e sintomas de abstinência após um período sem prática de exercícios.

Parastatidou et al. ${ }^{9}$ apontam que exercícios físicos podem ser considerados uma das 40 atividades com maior poten- cial de desenvolvimento de dependência/adição. Consequentemente, Hausenblas e Downs ${ }^{13}$ sugerem que, uma vez que a dependência esteja desenvolvida, a prática excessiva de exercício pode apresentar as seguintes consequências: irritabilidade, ansiedade, culpa e depressão, interferência no convívio familiar e social e no ambiente de trabalho, lesões musculoesqueléticas ou outras complicações devidas a cargas excessivas. Em uma direção oposta, Antunes et al. ${ }^{3}$ sugerem que, apesar de haver dependência de exercício, aparentemente isso não seria capaz de promover alterações significativas no estado de humor e na qualidade de vida dos corredores de aventura. Tais dados sugerem que alguns atletas apresentam dependência de exercício não associada aos distúrbios de humor.

A partir do exposto, é possível deduzir que as consequências da DEF são graves, a elaboração do seu diagnóstico é problemática e as poucas pesquisas relacionadas ao tema, no Brasil, contribuem para o desconhecimento por parte dos profissionais envolvidos com práticas esportivas. Portanto, desenvolver estudos nesse campo pode auxiliar profissionais na identificação da DEF, especialmente se forem disponibilizados instrumentos, adequados psicometricamente, que facilitem a identificação da dependência e, consequentemente, balizem intervenções apropriadas.

Portanto, tendo em conta o comentado anteriormente, o presente estudo busca contribuir nessa direção, ou seja, visa adaptar a Exercise Dependence Scale-Revised (EDS-R), construída por Downs et al. ${ }^{14}$, com base nos critérios de dependência de transtornos, relacionados ou não a substâncias, do DSM ${ }^{15}$. O critério para seleção dessa escala, em detrimento de outras escalas ${ }^{13,16,17}$, priorizou a adequação dos seus fatores ao seu modelo teórico, que buscou respaldo nos sintomas de dependência já constantes em manuais psiquiátricos de diagnóstico. Ademais, soma-se o fato de a EDS-R ser amplamente utilizada em contextos distintos, por exemplo, é possível identificar seu uso em estudos desenvolvidos em países como os Estados Unidos, Grécia, Hungria, Alemanha e Espanha ${ }^{1,6,7,18,19}$.

Downs et al. ${ }^{14}$ também argumentam que, para a identificação da dependência, é necessário que o instrumento de rastreamento contemple variáveis físicas e psicológicas e que seus itens estejam voltados para as implicações e prejuízos dessas variáveis no cotidiano dos indivíduos, critérios que, a medida de interesse, possuem em seu conteúdo. 
O instrumento é composto por 21 itens unidirecionais, respondidos numa escala de seis pontos, que variam de 1 (Nunca) a 6 (Sempre), distribuídos em sete fatores (três itens por fator), a serem descritos a seguir: (1) Tolerância/resistência, que se refere à necessidade que o indivíduo tem de aumentar sua carga de exercícios ou a sensação de que eles não estão fazendo mais efeito caso ele continue com a mesma carga anterior; (2) Evitar sintomas de abstinência, no qual o indivíduo se exercita para evitar irritabilidade e/ou ansiedade; (3) Intencionalidade, que se refere à prática de exercícios maior do que a planejada; (4) Falta de controle, definida a partir da incapacidade do indivíduo em reduzir sua carga de exercícios; (5) Tempo, representada por itens que expressam o grande tempo despendido em exercícios; (6) Redução de outras atividades, que se refere à diminuição do convívio social, ocupacional ou de lazer para se exercitar; e (7) Continuidade, que expressa a continuidade dos exercícios, mesmo quando eles estão contraindicados.

Na oportunidade de sua validação ${ }^{14}$, foram realizados dois estudos com procedimentos confirmatórios (análises fatoriais), nos quais a EDS-R apresentou índices satisfatórios de ajuste ao modelo teorizado. Especificamente, no estudo 1, foram observados os seguintes indicadores: $T L I=0,95 ; C F I$ $=0,96 ; \operatorname{RMSEA}=0,06 ; \mathrm{AASR}=0,03(p<0,05)$, enquanto no segundo estudo foi encontrado o seguinte ajuste dos dados ao modelo teórico: $\mathrm{TLI}=0,96 ; \mathrm{CFI}=0,97$; RMSEA =0,05; $\mathrm{AASR}$ =0,02, demonstrando evidências satisfatórias de sua validade de construto.

Diante do exposto, o presente estudo tem o objetivo geral de adaptar a EDS-R para o português brasileiro e verificar indícios de suas propriedades psicométricas para o contexto brasileiro. Nesse sentido, apresenta como objetivos específicos: (1) averiguar evidências preliminares de validade fatorial e consistência interna da EDS-R; (2) testar, por meio de análise fatorial confirmatória, sua estrutura fatorial e (3) apresentar dados preliminares de normatização da EDS-R.

\section{MÉTODOS}

\section{Amostra}

Para a efetivação dessa pesquisa, contou-se com a participação de 709 praticantes de exercício físico de diferentes estados do Brasil, distribuídos em duas amostras independentes (Amostras 1 e 2; pormenorizadamente descritas a seguir). Ambas foram não probabilísticas, isto é, de conveniência, tendo por critério de inclusão a prática de exercício físico, com duração mínima de 30 minutos e frequência igual ou superior a duas vezes por semana.

Amostra 1 - Compuseram a primeira amostra 333 praticantes regulares de exercício físico, com idades variando entre 14 e 55 anos ( $M=25,6$; DP = 8,34), em sua maioria do sexo masculino $(65,8 \%)$, solteiros $(81,1 \%)$, com renda entre 500 e 2.500 reais (33,0\%). Desses, 61,3\% indicaram não estar satisfeitos com o próprio corpo e praticar exercícios físicos em pelo menos cinco dias na semana (31,9\%), ademais afirmaram gastar um tempo médio de 60 minutos diários em exercícios físicos (34,2\%).

Amostra 2 - Para compor a segunda amostra do estudo, contou-se com 376 praticantes regulares de exercício físico, com idades variando entre 12 e 73 anos $(M=24,3 ; D P=7,56)$, em sua maioria do sexo masculino $(55,1 \%)$, solteiros $(75,5 \%)$, com renda entre 500 e 2.500 reais (33,7\%). Estes indicaram, em sua maioria, não estar satisfeitos com o próprio corpo (40,9\%), praticar exercícios físicos pelo menos cinco dias na semana (44,6\%), além de dispor, em média, 60 minutos diários para seus exercícios físicos (31,5\%).

\section{Instrumentos}

Os participantes responderam a um questionário composto por quatro partes. No entanto, em razão dos objetivos do presente estudo, apenas duas são aqui consideradas:

Exercise Dependence Scale-Revised (EDS-R). Essa medida, como previamente descrita, foi desenvolvida por Downs et al. ${ }^{14}$, em contexto estadunidense, para ser uma ferramenta de rastreio da Dependência de Exercício Físico (DEF), focada na avaliação dos prejuízos físicos e psicológicos e nas implicações desses prejuízos ao cotidiano dos indivíduos. A escala é composta por 21 itens distribuídos em sete fatores: (1) Tolerância/Resistência; (2) Evitar sintomas de abstinência; (3) Intencionalidade; (4) Falta de controle; (5) Tempo; (6) Redução de outras atividades; (7) Continuidade. Em contexto brasileiro, a medida foi traduzida e adaptada por Oliveira e Alchieri20, sendo respondida em uma escala de resposta do tipo Likert com extremos de 1 (Nunca) a 5 (Sempre).

Conforme propõem seus autores ${ }^{14}$, os escores da EDS-R podem ser interpretados tanto em formato intervalar quanto categorial. No primeiro caso, o escore total da EDS-R é interpretado enquanto um continuum (pontuação mínima 21 e máxima 105) no qual escores mais elevados indicam maior incidência de sintomas de DEF. Já a classificação categorial permite alocar os respondentes em três grupos, conforme as regras descritas a seguir: Indivíduos com pontuação médias acima de 4 em pelo menos três dos sete fatores são classificados como (1) Risco para Dependência de Exercício. Os participantes que endossam médias entre 3 e 4 ou uma combinação entre 3 e 4 e acima de 4 em pelo menos três dos sete fatores, desde que não cumpram os critérios de Risco para Dependência de Exercício, são classificados como (2) Não Dependente Sintomático. Já aqueles que apresentam médias abaixo dos 3 pontos em pelo menos três dos sete fatores, desde que não cumprem os critérios de Risco para Dependência de Exercício ou de Não Dependente Sintomático, são classificados como (3) Não Dependente Assintomático. 
Questões sociodemográficas - Além da EDS-R, foco do presente estudo, foram incluídas questões com o intuito de caracterização da amostra, a exemplo de perguntas sobre gênero, idade, estado civil, renda e profissão, bem como algumas versavam sobre hábitos relacionados à modificação corporal, como uso e consumo de suplementos ou medicamentos para alteração de peso, dietas etc.

\section{Procedimentos e aspectos éticos}

A coleta de dados se deu tanto em formato presencial, em academias, nas cidades de João Pessoa/PB e Natal/RN, quanto online, com a divulgação do link em sites de relacionamentos e fóruns de discussão sobre a temática.

Em ambos os contextos, após a explanação dos objetivos da pesquisa e o registro do consentimento livre e esclarecido dos participantes, foram repassadas instruções sobre o preenchimento individual do questionário, sendo assegurados o anonimato e a possibilidade de desistência a qualquer momento, sem qualquer tipo de prejuízo. O tempo médio para concluir a participação foi de aproximadamente 20 minutos. Essa pesquisa foi submetida e aprovada pelo Comitê de Ética em Pesquisa com Seres Humanos, da Universidade Federal de Natal (CEP/HUOL), sob o protocolo de $n^{\circ} 404 / 10$, sendo respeitados todos os princípios éticos para a realização de pesquisas com humanos, conforme a Resolução $n^{\circ}$ 466/12 do Conselho Nacional de Saúde.

\section{Análise de dados}

Os dados foram analisados por meio de procedimentos de análise fatorial exploratória e cálculo dos coeficientes alfas de Cronbach, utilizando-se, para tanto, o software IBM SPSS, versão 20. Posteriormente, empregando técnicas da Modelagem por Equação Estrutural (Structural Equation Modeling - SEM), por meio do software AMOS (versão 20), buscou-se conhecer a adequabilidade da solução fatorial encontrada. Nesse caso, considerou-se a matriz de covariância, tendo sido adotado o estimador ML (Maximum Likelihood).

Os seguintes indicadores de ajuste foram considerados como parâmetros de bondade de ajuste21-23:

(1) Razão $\chi^{2} / g$ l (qui-quadrado/grau de liberdade), admitindo-se valores entre 2 e 5 como indicativo de ajustamento adequado;

(2) Goodness-of-Fit Index (GFI), cujos valores variam de 0 a 1 , com aqueles próximos ou superiores a 0,90 indicando um ajustamento satisfatório do modelo teórico na explicação da matriz de variância-covariância;

(3) Comparative Fit Index (CFI), índice comparativo, adicional, de ajuste ao modelo, com valores mais próximos de 1 indicando melhor ajuste, tendo valor mínimo de 0,90 como referência para aceitar o modelo; e

(4) Root-Mean-Square Error of Approximation (RMSEA), refere-se ao ajuste entre o modelo teórico esperado e os dados empíricos obtidos, quanto mais próximo esse índice for de zero, melhor; contudo, admitem-se valores de até 0,10, levando em conta, geralmente, o ponto de corte de 0,08.

Adicionalmente, visando ampliar as evidências acerca da validade de construto da escala ${ }^{24,25}$, foram calculadas a variância média extraída (VME) e a confiabilidade composta (CC).

\section{RESULTADOS}

\section{Evidências de validade fatorial e consistência interna da EDS-R}

Nesta oportunidade, foi considerada a primeira amostra do estudo (amostra 1). Inicialmente, buscou-se conhecer o poder discriminativo dos itens que compõem a escala, considerando como critério a mediana empírica da pontuação total na medida. Por meio de uma MANOVA, observou-se que a variável critério discriminou satisfatoriamente o conjunto de itens [Lambda de Wilks $=0,34, F(21,300)=27,51, p<0,001$, $\left.\eta_{p}^{2}=0,66\right]$. Adicionalmente, os testes univariados (efeito entre sujeitos) demonstraram que os 21 itens discriminaram satisfatoriamente $(p<0,001)$ os dois grupos, não sendo indicada, assim, a exclusão de nenhum deles.

Com esse aspecto assegurado e garantida a fatorabilidade da matriz de dados $[K M O=0,85$; Teste de Esfericidade de Bartlett $\left.=\chi^{2}(210)=3450,90, p<0,001\right]$, passou-se para a próxima etapa, na qual os dados foram submetidos aos procedimentos da análise fatorial exploratória. O método de extração utilizado foi o de análise dos fatores comuns (principal axis factoring), com rotação ortogonal (varimax). O número de fatores foi fixado em 7 (sete), com base na proposta teórica dos autores originais da escala ${ }^{14}$. A composição fatorial proposta foi capaz de explicar $62,70 \%$ da variância total, tendo alcançado, ainda, bons índices de consistência interna (Tabela 1).

Com o intuito de interpretar as dimensões que compõem a escala, considerou-se que o item deveria apresentar saturação mínima (carga fatorial), no fator a qual pertence, igual ou superior a $|0,40|$. Com base nessa análise, foram calculados os coeficientes de consistência interna (alfa de Cronbach) por fator, os quais variaram entre 0,68 e 0,89, tendo a escala total alcançado um a de 0,88 , como pode ser observado na tabela 1. Ressalta-se que na descrição e interpretação de cada um dos fatores, apresentada a seguir, buscou-se seguir a nomenclatura adotada na versão original ${ }^{14}$.

Fator I - Intencionalidade. Refere-se à prática de exercício físico (EF) superior à sua intenção de fazê-lo. Esse fator reuniu 3 (três) itens, com saturações variando entre 0,70 (item 7) e 0,81 (item 14), que explicaram 11,38\% da variância total e obtiveram índices de consistência interna de 0,87;

Fator II - Continuidade. Este fator representa uma continuidade dos exercícios, mesmo quando são contraindicados. Ficou composto por 3 (três) itens [saturações entre 0,79 (item 16) e 0,80 (item 2 e 9)], explicando 10,67\% da variância total. Seu índice de consistência interna foi meritório $(a=0,89)$; 
Tabela 1. Estrutura fatorial da Exercise Dependence Scale-Revised

\begin{tabular}{|c|c|c|c|c|c|c|c|c|c|c|}
\hline \multirow{2}{*}{ Itens } & \multicolumn{7}{|c|}{ Fatores } & \multirow{2}{*}{$h^{2}$} & \multirow{2}{*}{$r_{i t}$} & \multirow{2}{*}{$a_{\text {del }}$} \\
\hline & 1 & II & III & IV & V & VI & VII & & & \\
\hline Item 14 & 0,81 & 0,14 & 0,11 & 0,09 & 0,17 & 0,14 & 0,20 & 0,80 & 0,63 & 0,88 \\
\hline Item 7 & 0,70 & 0,15 & 0,18 & 0,10 & 0,04 & 0,08 & 0,21 & 0,62 & 0,56 & 0,88 \\
\hline Item 21 & 0,75 & 0,12 & 0,11 & 1,12 & 0,17 & 0,11 & 0,22 & 0,71 & 0,67 & 0,88 \\
\hline Item 2 & 0,08 & 0,80 & 0,10 & 0,17 & 0,05 & 0,21 & 0,14 & 0,76 & 0,55 & 0,88 \\
\hline Item 16 & 0,15 & 0,79 & 0,06 & 0,13 & 0,07 & 0,13 & 0,06 & 0,70 & 0,51 & 0,88 \\
\hline Item 9 & 0,19 & 0,80 & 0,14 & 0,17 & 0,02 & 0,13 & 0,06 & 0,76 & 0,56 & 0,88 \\
\hline Item 10 & 0,10 & 0,11 & 0,78 & 0,12 & 0,21 & 0,11 & 0,17 & 0,74 & 0,53 & 0,88 \\
\hline Item 3 & 0,11 & 0,10 & 0,52 & 0,29 & 0,34 & 0,00 & 0,05 & 0,49 & 0,50 & 0,88 \\
\hline Item 17 & 0,25 & 0,11 & 0,64 & $-0,07$ & 0,01 & 0,22 & 0,11 & 0,56 & 0,47 & 0,88 \\
\hline Item 19 & 0,13 & 0,20 & 0,05 & 0,81 & 0,17 & 0,05 & 0,17 & 0,79 & 0,56 & 0,88 \\
\hline Item 5 & 0,06 & 0,16 & 0,08 & 0,85 & 0,15 & 0,09 & 0,08 & 0,79 & 0,50 & 0,88 \\
\hline Item 12 & 0,20 & 0,17 & 0,08 & 0,56 & 0,32 & 0,04 & 0,18 & 0,53 & 0,56 & 0,88 \\
\hline Item 11 & 0,11 & 0,06 & 0,05 & 0,09 & 0,86 & 0,07 & 0,13 & 0,80 & 0,46 & 0,88 \\
\hline Item 18 & 0,17 & 0,06 & 0,13 & 0,20 & 0,59 & $-0,03$ & 0,07 & 0,45 & 0,42 & 0,88 \\
\hline Item 4 & 0,02 & $-0,00$ & 0,11 & 0,11 & 0,59 & 0,05 & 0,03 & 0,38 & 0,31 & 0,89 \\
\hline Item 15 & 0,16 & 0,07 & 0,03 & 0,02 & 0,00 & 0,88 & 0,00 & 0,81 & 0,35 & 0,88 \\
\hline Item 1 & 0,05 & 0,23 & 0,08 & 0,11 & $-0,04$ & 0,62 & 0,06 & 0,46 & 0,36 & 0,89 \\
\hline Item 8 & 0,04 & 0,04 & 0,10 & 0,01 & 0,10 & 0,42 & 0,08 & 0,21 & 0,27 & 0,90 \\
\hline Item 20 & 0,40 & 0,11 & 0,13 & 0,16 & 0,10 & 0,09 & 0,70 & 0,73 & 0,60 & 0,88 \\
\hline Item 13 & 0,19 & 0,09 & 0,13 & 0,17 & 0,16 & 0,14 & 0,68 & 0,65 & 0,58 & 0,88 \\
\hline Item 6 & 0,35 & 0,18 & 0,21 & 0,16 & 0,08 & 0,02 & 0,43 & 0,42 & 0,53 & 0,88 \\
\hline Número de itens & 3 & 3 & 3 & 3 & 3 & 3 & 3 & - & - & - \\
\hline Valor próprio empírico & 2,39 & 2,24 & 1,54 & 2,08 & 1,89 & 1,57 & 1,44 & - & - & - \\
\hline Variância explicada & 11,38 & 10,67 & 7,35 & 9,90 & 9,01 & 7,48 & 6,89 & - & - & - \\
\hline Alfa de Cronbach & 0,87 & 0,89 & 0,74 & 0,84 & 0,75 & 0,68 & 0,75 & - & - & - \\
\hline
\end{tabular}

$h^{2}$ : comunalidade, $r_{i t i}$ correlação item-total, $a_{\text {del }}$ : alfa de Cronbach se 0 item fosse deletado.

Fator III - Tolerância. Este termo, utilizado no campo fisiológico, refere-se à necessidade de aumentar a carga de EF para sentir-se satisfeito. Tal fator reuniu 3 (três) itens, explicando 7,35\% da variância total, com itens que apresentaram saturações variando entre 0,52 (item 3) e 0,78 (item 10), quanto a precisão, apresentou a de 0,74.

Fator IV - Redução de outras atividades. Esta dimensão diz respeito à redução do convívio com familiares e amigos para passar tempo praticando EF. Assim como os anteriores, reuniu três itens com cargas fatoriais variando entre 0,56 (item 12) e 0,81 (item 5), que explicaram $9,90 \%$ da variância total, com consistência interna adequada $(a=0,84)$.

Fator V - Falta de controle. Este fator indica uma falta de controle do indivíduo na prática de EF, tendo que manter sempre a intensidade, duração e frequência do exercício para se sentir satisfeito. A exemplo dos demais, também foi composto por três itens, os quais apresentaram saturações entre 0,59 (item 4 e 18) e 0,86 (item 11). Esta dimensão explicou 9,01\% da variância e consistência interna de 0,75;
Fator VI - Abstinência. Propõe que, quando privado de se exercitar, o indivíduo passa a sentir sintomas característicos de abstinência, como ansiedade, irritabilidade e tensão. Agrupou três itens e sua variância explicada foi de 7,48, com cargas fatoriais variando entre 0,42 (item 8) e 0,88 (item 15) e consistência interna satisfatória $(a=0,68)$;

Fator VII - Tempo. Refere-se ao tempo despendido pelo indivíduo na prática de EF. Este fator, composto por três itens, explicou 6,89\% da variância, apresentando itens com cargas entre 0,43 (item 6) e 0,70 (item 20), ademais, seu alfa de Cronbach foi aceitável $(a=0,79)$.

Em resumo, esses resultados parecem evidenciar parâmetros psicométricos aceitáveis da Exercise Dependence Scale. Contudo, reconhecendo o caráter essencialmente exploratório das técnicas estatísticas empregadas e a ausência de estudos em contexto brasileiro que tenham empregado a medida, faz-se necessário conhecer em que medida a estrutura heptafatorial é replicável em uma amostra independente. Aspecto que motivou ter em conta técnicas mais 
robustas, a exemplo da análise fatorial confirmatória (CFA), descrita a seguir.

\section{Análise fatorial confirmatória da EDS-R}

Para testar a estrutura multidimensional previamente descrita para a EDS-R, efetuou-se uma Análise Fatorial Confirmatória (CFA), considerando a matriz de covariância e empregando o estimador Maximum Likelihood. Nesse caso, teve-se em conta a segunda amostra do estudo (amostra 2).

Com base nos resultados encontrados, foi possível verificar que os indicadores de bondade de ajuste do modelo revelaram uma solução satisfatória: $\chi^{2}(168)=288,21 ; p<0,001$, $\chi^{2} / \mathrm{gl}=1,78, \mathrm{GFI}=0,93, \mathrm{CFI}=0,95$ e RMSEA $=0,04(\mathrm{IC} 90 \%=$ $0,04-0,05)$. Todos os itens da escala apresentaram saturações (pesos fatoriais, $\lambda$ ) estatisticamente diferentes de zero $(\lambda$ $\neq 0 ; z>1,96, p<0,05)$. A estrutura correspondente pode ser observada na figura 1.

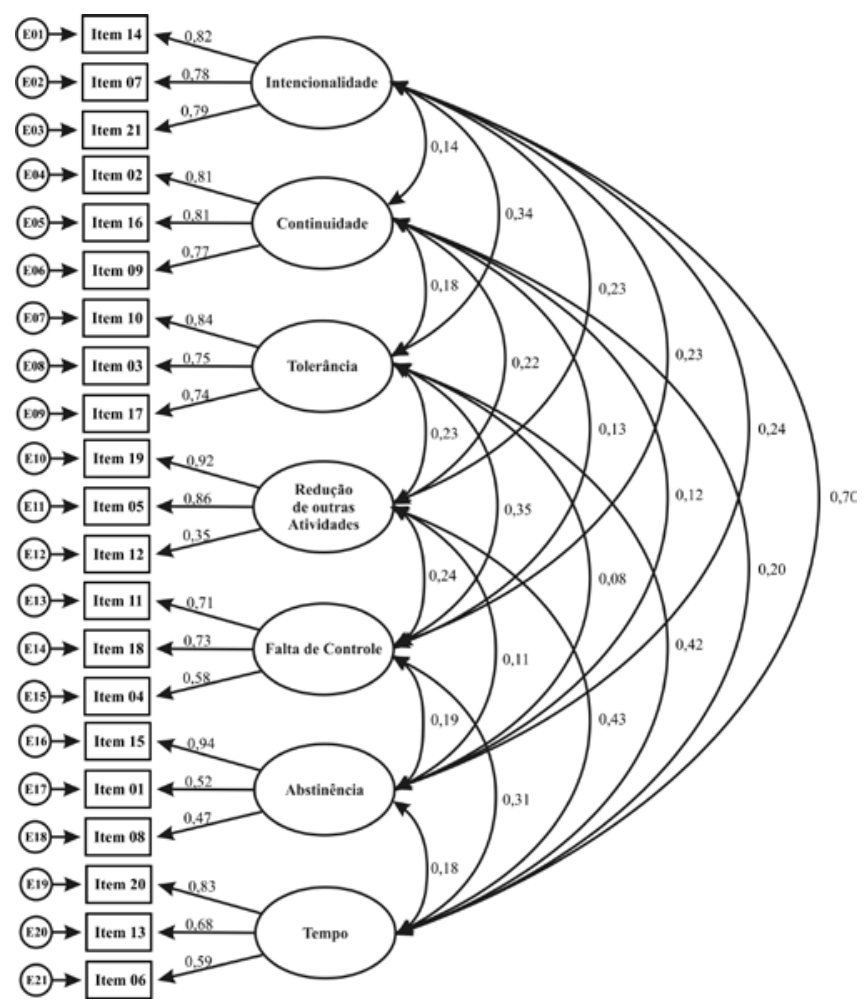

Figura 1. Modelo da estrutura fatorial da Exercise Dependence Scale-Revised.

Comprovados a pertinência e o ajuste aceitável do modelo heptafatorial, procurou-se, em seguida, reunir evidências complementares de sua validade de construto e precisão. No que consiste a sua confiabilidade composta (Tabela 2), os valores encontrados foram superiores ao recomendado pela literatura $\left(0,70\right.$; ver Hair et al. $\left.{ }^{25}\right)$. No caso da variância média extraída, sua validade convergente ficou também evidenciada ${ }^{25}$.
Tabela 2. Precisão e validade convergente dos fatores da Exercise Dependence Scale-Revised

\begin{tabular}{lccc}
\hline Fator & $\boldsymbol{a}$ & $\boldsymbol{c C}$ & JVME \\
\hline Intencionalidade & 0,84 & 0,90 & 0,79 \\
Continuidade & 0,84 & 0,90 & 0,80 \\
Tolerância & 0,81 & 0,89 & 0,78 \\
Redução & 0,73 & 0,84 & 0,75 \\
Falta de controle & 0,71 & 0,81 & 0,67 \\
Abstinência & 0,66 & 0,78 & 0,67 \\
Tempo & 0,73 & 0,83 & 0,71 \\
\hline
\end{tabular}

a: Alfa de Cronbach, CC: Confiabilidade Composta, JVME: Raiz Quadrada da Variância Média Extraída.

Como se pode verificar, em termos gerais, os procedimentos de análise fatorial confirmatória atestaram a adequabilidade do modelo de sete fatores, corroborando, assim, a solução fatorial apresentada anteriormente (amostra 1), o que é evidenciado pelos bons índices de fidedignidade alcançados. Por fim, confiante nas qualidades psicométricas do instrumento hora adaptado e a fim de garantir a interpretabilidade dos escores brutos obtidos, optou-se por realizar a normatização preliminar da EDS-R.

\section{Normatização preliminar da EDS-R}

Os parâmetros para interpretação dos resultados foram calculados baseados no posto percentílico e na determinação dos escores-padrão normatizados (escore T) dos 709 participantes que compuseram a amostra total da pesquisa (amostras 1 e 2).

As tabelas normativas foram elaboradas considerando o gênero dos participantes, uma vez que as médias de dependência de exercício foram significativamente $(t=5,28 ; p<$ $0,001)$ diferentes para homens $(M=57,9 ; D P=12,3)$ e muIheres $(M=52,8 ; D P=11,8)$. Por essa razão, foram elaboradas normas distintas para cada gênero, como pode ser visualizado na tabela 3.

Não obstante os resultados ora apresentados, deve-se considerar que, para fins clínicos, é imprescindível que a escala apresente uma definição clara de como os profissionais devem interpretar seus resultados. Para tanto, os autores da versão original da escala ${ }^{14}$ propuseram critérios de classificação para a dependência de exercício físico com base na pontuação média por fator (para maior detalhamento, ver subtópico descrição da EDS-R), agrupando os respondentes em três grupos distintos. A fim de testar a adequabilidade da classificação proposta, realizou-se uma Análise de Variância (ANOVA) comparando as pontuações médias na EDS-R obtidas pelos respondentes alocados em cada um dos grupos.

Como visualizado na tabela 4, os resultados indicaram diferenças estatisticamente significativas entre os grupos [ $F$ $(2 ; 663)=306,18 ; p<0,001]$. Especificamente, o post hoc de Bonferroni mostrou que os respondentes classificados como de Risco para Dependência de Exercício apresentaram, como esperado, maiores pontuações totais em DEF $(M=79,4 ; D P$ 
=9,84), quando comparados ao Não Dependente Sintomático $(M=60,8 ; D P=8,53)$ e ao Não Dependente Assintomático ( $M$ $=48,6 ; D P=9,17)$.

Tabela 3. Normas percentílicas e escores T para gênero e amostra total $(N=709)$

\begin{tabular}{|c|c|c|c|c|c|c|}
\hline \multirow{3}{*}{ Percentil } & \multicolumn{4}{|c|}{ Gênero } & \multirow{2}{*}{\multicolumn{2}{|c|}{ Amostra total }} \\
\hline & \multicolumn{2}{|c|}{ Masculino } & \multicolumn{2}{|c|}{ Feminino } & & \\
\hline & $\begin{array}{l}\text { Escore } \\
\text { Bruto }\end{array}$ & Escore T & $\begin{array}{l}\text { Escore } \\
\text { Bruto }\end{array}$ & Escore T & $\begin{array}{l}\text { Escore } \\
\text { Bruto }\end{array}$ & Escore T \\
\hline 1 & 33 & 30 & 29 & 30 & 32 & 31 \\
\hline 5 & 39 & 35 & 35 & 35 & 37 & 35 \\
\hline 10 & 43 & 38 & 38 & 37 & 41 & 38 \\
\hline 15 & 45 & 39 & 40 & 39 & 43 & 40 \\
\hline 20 & 47 & 41 & 43 & 42 & 45 & 41 \\
\hline 25 & 49 & 43 & 44 & 42 & 46 & 42 \\
\hline 30 & 51 & 44 & 45 & 43 & 49 & 44 \\
\hline 40 & 54 & 47 & 50 & 48 & 52 & 47 \\
\hline 50 & 57 & 49 & 52 & 49 & 56 & 50 \\
\hline 60 & 60 & 52 & 56 & 53 & 59 & 52 \\
\hline 70 & 63 & 54 & 58 & 54 & 61 & 54 \\
\hline 75 & 66 & 56 & 60 & 57 & 64 & 56 \\
\hline 80 & 67 & 57 & 63 & 58 & 66 & 58 \\
\hline 85 & 70 & 60 & 64 & 59 & 68 & 60 \\
\hline 90 & 74 & 63 & 68 & 63 & 72 & 63 \\
\hline 95 & 81 & 69 & 73 & 67 & 79 & 69 \\
\hline 99 & 92 & 78 & 89 & 80 & 89 & 78 \\
\hline
\end{tabular}

Tabela 4. Comparação de médias na pontuação total da Exercise Dependence Scale-Revised entre os distintos grupos de classificação $(N=709)$

\begin{tabular}{lccccc}
\hline Classificação & N & M & DP & F & P \\
\hline Risco para Dependência de Exercício & 44 & 79,4 & 9,84 & & \\
Não Dependente Sintomático & 288 & 60,8 & 8,53 & 306,18 & $<0,001$ \\
Não Dependente Assintomático & 334 & 48,6 & 9,17 & & \\
\hline
\end{tabular}

\section{DISCUSSÃO}

A perspectiva teórica que fundamentou o presente artigo foi a defendida por Downs et al. ${ }^{14}$, em razão de estar fundamentada em um manual diagnóstico amplamente aceito ${ }^{15}$, contemplando dimensões voltadas para as implicações psicológicas, fisiológicas e sociais da dependência.

O procedimento escolhido para avaliar a adequação do modelo empírico ao modelo teórico foi a análise fatorial, que é um método multivariado para definir uma estrutura subjacente em uma matriz de dados ${ }^{26}$. Os resultados apontaram para uma estrutura de sete fatores, com três descritores em cada um, consonante com o modelo teórico, sendo, portanto, referendada nas amostras aqui utilizadas.
Quanto aos índices de consistência interna, eles foram avaliados como meritórios se consideradas homogeneidade dos itens, consistência interna, dos fatores e escala total, além da confiabilidade composta. Além do mais, é possível ressaltar a pertinência das evidências de precisão quando se tem em conta o número de itens nas subescalas, a natureza do constructo e o conteúdo dos itens ${ }^{27}$.

Quanto ao emprego da SEM, observou-se que os índices de bondade de ajuste do modelo, encontrados na análise fatorial confirmatória, apresentaram-se satisfatórios, de acordo com os critérios de Byrne ${ }^{21}$, Garson ${ }^{22}$ e Tabachnick e Fidell ${ }^{23}$. A razão $\chi^{2} / g$ I indicou um ajuste satisfatório, apresentando valor sensivelmente inferior ao recomendado, isto é, 521,23. Outros indicadores de bondade foram na mesma direção, indicando a adequação do modelo teorizado por Downs et al. 14; são os casos do GFI e CFI, cujos valores foram superiores a 0,90, e o RMSEA, que se apresentou inferior a 0,08, valores, esses tidos como referência ${ }^{22}$.

Os índices psicométricos e a estrutura fatorial encontrados nesse estudo, seja por procedimento exploratório ou confirmatório, estão em conformidade com aqueles sugeridos pelos autores originais da escala ${ }^{14}$, bem como por outros estudos em contexto internacional6,18,19,28.

Os resultados aqui obtidos foram consonantes com os alcançados por Parastatidou et al. ${ }^{28}$, quando da validação da EDS-R para o contexto grego, por meio de análises fatoriais confirmatórias, que apontaram a pertinência do modelo com sete fatores. Ademais, Mónok et al. ${ }^{6}$ também observaram essa estrutura em 474 praticantes de exercícios físicos na Hungria, com índices de bondade de ajuste do modelo similares aos observados nesse estudo ( $C F I=0,93 ; \mathrm{TLI}=0,92$; RMSEA = 0,04).

Sicilia e González-Cutre ${ }^{18}$, por sua vez, realizaram a validação da EDS-R para o contexto espanhol, utilizando-se de uma amostra de 531 praticantes de exercícios físicos, e confirmaram o modelo teórico proposto originalmente. Ainda que tendo observado sete fatores de primeira ordem e um único fator de segunda ordem (dependência do exercício físico) com indicadores psicométricos satisfatórios, os fatores estão em consonância com aqueles encontrados aqui.

Após a apresentação dos índices psicométricos da escala, optou-se por delinear parâmetros preliminares de normatização dos dados, com vistas a possibilitar uma interpretação mais adequada do instrumento. Previamente à elaboração dos dados percentílicos, procedeu-se a um teste de diferenciação de médias, para verificar se os dados variavam em função do sexo dos participantes.

Considerando que os resultados encontrados apontaram para escores médios significativamente diferentes de dependência de exercício em homens e mulheres, procedeu-se à elaboração da tabela normativa para as duas populações. Esses achados são contrastantes com aqueles apresentados por Rosa et al. ${ }^{2}$, Antunes et al. ${ }^{3}$, Duarte ${ }^{29}$ e Vieira et al. ${ }^{30}$, os 
quais não observaram diferenciação de escores em DEF em função do gênero.

Em contrapartida, os estudos de Pierce et al. ${ }^{31}$ revelaram diferenças entre os gêneros, considerando que homens apresentam maiores sentimentos de desconforto quando interrompem seus programas de treinamento. Edmundus et al. ${ }^{32}$ constataram escores mais elevados de dependência de exercícios em homens, diferença essa considerada significativa quando comparados aos escores femininos. Esses mesmos autores constataram que indivíduos com sintomas de dependência de exercício apresentaram escores mais elevados de automotivação (ou motivação intrínseca) do que aqueles que não possuem sintomas.

\section{CONCLUSÃO}

O presente estudo objetivou verificar evidências de validade da EDS-R para o contexto nacional, e os resultados encontrados apontaram para parâmetros favoráveis e satisfatórios do instrumento, corroborando o modelo teórico vigente, bem como apontando para o uso adequado do instrumento.

Como sugestão para pesquisas futuras, destacam-se replicações deste estudo, com vistas a observar a disposição da estrutura fatorial e manifestação da DEF em outras amostras. Sugere-se, ainda, a observação da DEF e sua relação, seja como variável antecedente ou consequente de outros fenômenos, a exemplo de comprometimento psicológico com o hábito de se exercitar, lócus de controle, teoria da autodeterminação, motivações e tipos de exercícios físicos.

\section{CONTRIBUIÇÕES INDIVIDUAIS}

João Carlos Alchieri - Contribuiu significativamente na análise e interpretação dos dados, revisou criticamente o seu conteúdo intelectual e aprovou sua versão final a ser publicada.

Valdiney Veloso Gouveia - Contribuiu significativamente na análise e interpretação dos dados, revisou criticamente o seu conteúdo intelectual e aprovou sua versão final a ser publicada.

Isabel Cristina Vasconcelos de Oliveira - Contribuiu significativamente na concepção e desenho do estudo, contribuiu substancialmente na elaboração do artigo e aprovou sua versão final a ser publicada.

Emerson Diógenes de Medeiros - Contribuiu significativamente na análise e interpretação dos dados, revisou criticamente o seu conteúdo intelectual e aprovou sua versão final a ser publicada.

Alex Sandro de Moura Grangeiro - Contribuiu significativamente na concepção e desenho do estudo, contribuiu substancialmente na elaboração do artigo e aprovou sua versão final a ser publicada.

Carla Fernanda de Lima Santiago da Silva - Contribuiu significativamente na concepção e desenho do estudo, contribuiu substancialmente na elaboração do artigo e aprovou sua versão final a ser publicada.

\section{CONFLITOS DE INTERESSE}

Os autores não possuem conflitos de interesse a serem declarados.

\section{AGRADECIMENTOS}

Assistência obtida da fonte de financiamento Coordenação de Aperfeiçoamento Pessoal de Nível Superior (CAPES), por meio de bolsa do Programa de Demanda Social (DS), nível Mestrado.

\section{REFERÊNCIAS}

1. Lichtenstein MB, Christiansen E, Elklit A, Bilenberg N, Støving RK. Exercise addiction: a study of eating disorder symptoms, quality of life, personality traits and attachment styles. Psychiatry Res. 2014;215(2):410-6.

2. Rosa DA, Mello MT, Souza-Formigoni MLO. Dependência da prática de exercícios físicos: estudo com maratonistas brasileiros. Rev Bras Med Esporte. 2003;9(1):9-14.

3. Antunes HKM, Andersen ML, Tufik S, Mello MT. 0 estresse físico e a dependência de exercício físico. Rev Bras Med Esporte. 2006;12(5):234-8.

4. Modolo VB, Mello MT, Gimenez PRB, Tufik S, Antunes HKM. Dependência de exercício físico: humor, qualidade de vida em atletas amadores e profissionais. Rev Bras Med Esporte. 2009;15(5):355-9.

5. Soler PT, Fernandes HM, Damasceno VO, Novaes JS. Vigorexia e níveis de dependência de exercício em frequentadores de academias e fisiculturistas. Rev Bras Med Esporte. 2013;19(5):343-8.

6. Mónok K, Berczik K, Urbán R, Szabo A, Griffiths MD, Farkas J. Psychometric properties and concurrent validity of two exercise addiction measures: a population wide study. Psychol Sport Exercise. 2012;13:739-46.

7. Paradis KF, Cooke LM, Martin LJ, Hall CR. Too much of a good thing? Examining the relationship between passion for exercise and exercise dependence. Psychol Sport Exercise. 2013;14:493-500.

8. Stenseng F, Haugen T, Torstveit MK, Hoigaard R. When it's "all about the bike" - intrapersonal conflict in light of passion for cycling and exercise dependence. Sport Exercise Perform Psychol. 2015;4(2):127-39.

9. Parastatidou IS, Doganis G, Theodorakis Y, Vlachopoulos SP. The mediating role of passion in the relationship of exercise motivational regulations with exercise dependence symptoms. Int J Ment Health Addict. 2014;12:406-19.

10. Smith D, Hale B. Validity and factor structure of the bodybuilding dependence scale. Br J Sports Med. 2004;38(2):177-81.

11. Márquez $S$, de la Vega R. La adicción al ejercicio: un trastorno emergente de la conducta. Nutrición Hospitalaria. 2015;31(6):2384-91.

12. Griffiths MD. Exercise addiction: a study case. Addict Res Theory. 1997;5:161-8.

13. Hausenblas HA, Downs DS. How much is too much? The development and validation of the exercise dependence scale. Psychol Health. 2002;17:387-404. 
14. Downs DS, Hausenblas HA, Niggs CR. Factorial validity and psychometric examination of the exercise dependence scale-revised. Meas Phys Educ Exerc Sci. 2004;8:183-201.

15. American Psychiatric Association. Manual Diagnóstico e Estatístico de Transtornos Mentais. Porto Alegre: Artmed; 1994.

16. Ogden J, Veale D, Summers $Z$. The development and validation of the exercise dependence questionnaire. Addict Res. 1997;5:343-56.

17. Terry A, Szabo A, Griffiths $M$. The exercise addiction inventory: a new brief screening tool. Addict Res Theory. 2004;12:489-99.

18. Sicilia A, González-Cutre D. Dependence and physical exercise: Spanish validation of the Exercise Dependence Scale-Revised (EDS-R). Span J Psychol. 2011;14(1):421-31.

19. Mueller A, Claes L, Smits D, Gefeller O, Hilbert A, Herberg, A, et al. Validation of the German version of the exercise dependence scale. Eur J Psychol Assess. 2013;29(3):213-9.

20. Oliveira ICV, Alchieri JC. Tradução e análise teórica da escala de dependência de exercício físico. FIEP Bull. 2010;80:1-6

21. Byrne B. Structural equation modeling with AMOS. Basic concepts, applications and programming. Mahwah: Lawrence Erlbaum Associates; 2001.

22. Garson GD. PA 765 Statnotes: An online textbook. Disponível em: <http://www2.chass. ncsu.edu/garson/pa765/statnote.htm>. Acesso em: 8 set. 2007

23. Tabachnick BG, Fidell LS. Using multivariate statistics. Nova York: Allyn \& Bacon; 2001
24. Fornell C, Larcker DF. Evaluating structural equation models with unobservable variables and measurement error. J Marketing Res. 1981;39-50.

25. Hair JF, Anderson RE, Tatham RL, Black WC. Análise multivariada de dados. Porto Alegre: Artmed; 2005

26. Dancey (P, Reidy J. Estatística sem matemática para Psicologia. Porto Alegre: Artmed; 2006.

27. Gouveia VV, Santos WS, Milfont TL. 0 uso da estatística na avaliação psicológica: comentários e considerações práticas. In: Hutz CS, editor. Avanços e polêmicas em avaliação psicológica. São Paulo: Casa do Psicólogo; 2009.

28. Parastatidou IS, Doganis G, Theodorakis Y, Vlachopoulos SP. Psychometric properties of the exercise dependence scale-revised in a sample of Greek exercise participants. Eur J Psychol Assess. 2012;28(1):3-10.

29. Duarte PJP. Dependência ao exercício em indivíduos frequentadores de ginásios [trabalho de conclusão de curso]. Graduação em Educação Física. Coimbra: Faculdade de Ciências do Desporto e Educação Física da Universidade de Coimbra; 2009.

30. Vieira JLL, Rocha PGM, Ferrarezzi RA. A dependência pela prática de exercícios físicos e 0 uso de recursos ergogênicos. Acta Scientiarum. Health Sci. 2010;32:35-41.

31. Pierce EF, Rohaly KA, Fritchley B. Sex differences on exercise dependence for men and women in a marathon road race. Percept Mot Skills. 1997:84(3 Pt 1):991-4.

32. Edmundus J, Ntoumanis N, Duda JL. Examining exercise dependence symptomatology from a self-determination perspective. J Health Psychol. 2006;11:887-903. 